\title{
Forest radiative transfer models: which approach for which application?
}

\author{
Gauthier Ligot ${ }^{1 *}$, Philippe Balandier ${ }^{2}$, Benoît Courbaud ${ }^{3}$, Hugues Claessens ${ }^{1}$
}

\begin{abstract}
Radiation is fundamental in forest ecosystem ecology as it drives plant photosynthesis, morphogenesis, and fluxes of carbon, water and energy between soil, vegetation, and the atmosphere. Though all approaches of forest radiative transfer models (FRTM) share general properties, they differ greatly in terms of calibration parameters, required assumptions, and model objectives. They use different precision levels of canopy description (from one to three dimensions) and different mathematical relationships to model the attenuation of radiation through the canopy. To date no general guideline has been given to help the modeler choose the approach that best suits his needs. We therefore reviewed evaluation, sensitivity, and performance of FRTMs recently reported in the literature. We quantified FRTM uncertainty and identified the most sensitive parameters relative to the modeling choices. Their advantages and drawbacks are discussed and recommendations are made relative to application potential.

${ }^{1}$ Univ. de Liège, Gembloux Agro-Bio Tech, Unité de Gestion des Ressources forestières et des Milieux naturels, B-5030 Gembloux, Belgium

${ }^{2}$ Irstea, U.R. Ecosystèmes Forestiers (EFNO), Domaine des Barres, 45290 Nogent-sur-Vernisson, France

${ }^{3}$ Irstea, Mountain Ecosystems Research Unit, 2 rue de la Papeterie, F-38402 Saint Martin d'Héres, France

*Corresponding author: gligot@ulg.ac.be
\end{abstract}

\section{Contents}

\section{Introduction}

2 Classification of FRTMs

2.1 Canopy geometry . . . . . . . . . . . . . . . 2 One-dimensional canopy $\bullet$ 3D crown models $\bullet 3 D$ surface models

2.2 Radiation attenuation . . . . . . . . . . . . 4 Turbid medium • Porous envelope

3 Input variables 5

3.1 Stand and tree measurements $\ldots \ldots \ldots \ldots$

3.2 Density of canopy elements . . . . . . . . 6

3.3 Extinction coefficients $\ldots \ldots \ldots \ldots$

3.4 Clumping factors $\ldots \ldots \ldots \ldots$

3.5 Crown openness . . . . . . . . . . . . 7

4 Model applications and output variables 7

5 Model evaluation

6 Model sensitivity

6.1 1D model . . . . . . . . . . . . . . . . 9

6.2 3D crown model . . . . . . . . . . . . . . . 12

7 Discussion

12

7.1 Model uncertainty . . . . . . . . . . . . . . . . 12

7.2 Sensitive input parameters . . . . . . . . . . . . . . . . . . . . . . . . . .

7.3 Applications . . . . . . . . . . . . . . . . 14

\section{Introduction}

Radiation is fundamental in forest ecology. It drives plant photosynthesis and exchanges of masses and energy between soil, vegetation, and atmosphere. As a consequence, the amount of energy and the spectrum of the radiation intercepted by trees have been widely studied as they affect greatly the gross primary production of forests (Duursma and Mäkelä, 2007; Tian et al., 2010), forest dynamics and competition between species (Pacala et al., 1996), individual tree growth (Balandier et al., 2007; Lieffers et al., 1999), and the morphogenesis of stems, branches, leaves, and root systems (Balandier et al., 2006; Galen et al., 2007; Niinemets, 2010).

Measuring the amount of radiations, and particularly Photosynthetically Active Radiation (PAR), intercepted by the diverse components of a forest canopy is tedious and timeconsuming. Compare with PAR measurements in standard agricultural crops, and due to the complexity of the forest canopy with different strata, the spatial and temporal variability of transmitted light beneath the forest canopy is substantial and vegetation beneath a closed canopy can benefit from sunflecks or very brief increases of transmitted irradiance (Messier et al., 1999). Measurements must then be repeated in numerous points and for rather long periods of time to catch this variability. Moreover, measuring transmitted PAR at various canopy heights is almost impossible without installing a crane (Mariscal et al., 2004). Face to these difficulties and to the cost of such measurements, modeling radiative transfer appears essential to supplement the field measurements and to better understand how radiations are shared between the components of forest ecosystem.

Solar radiation and the attenuation of light through the plant canopy were first modeled with physical laws by analogy of the Beer's law in homogeneous medium. Consequently, they were firstly limited to homogeneous crops but were 
rapidly adapted to forest ecosystems using more complex laws. The general functioning of these latter models have already been presented in several literature reviews (Brunner, 1998; Lieffers et al., 1999; Sinoquet et al., 1993) and have changed little since then. Briefly, they share a common structure that can be divided into three submodels (Fig. 1). Firstly, most models start by computing above canopy light both in magnitude and angular distribution using standard astronomical laws ("above- canopy light model"). Secondly, the interception of light through the canopy ("radiative transfer model") is computed. It depends mainly on the geometric structure of the canopy and the mathematical formulation used to describe the fractions of light that are intercepted, absorbed, and transmitted by canopy components. Thirdly, a light reflection model can optionally be used ("scattering model") to describe more precisely complex light trajectories within the canopy.

Even though most forest radiative transfer models (FRTMs) share a common general framework, they have special features that rely on different assumptions and they are used to predict different output variables aggregated or not in time and space according to the objectives of the model. The costs of specific field measurements to calibrate the model, the increasing interest in complex forests composed of trees of different species and sizes, and the variety of study purposes have indeed stimulated modelers to adapt FRTMs to their needs. Most of these adaptations concerned the radiative transfer model, one of the submodels depicted in figure 1 , which is the main topic of this review. For example, contrasted approaches have been used to model the distribution of foliage within the canopy. Some authors have used very detailed three-dimensional (3D) mock-ups of the canopy, which require intensive field measurements, whereas others describe the canopy as a single horizontal layer. The improvements brought to above canopy light models are very limited as only two standard algorithms (uniform overcast sky or standard overcast sky) have been used among all off the papers that we reviewed. Moreover, the use of one of them does not really affect model performance (Brunner, 1998), i.e. model ability to predict the measurements with a low level of error and bias. Similarly, the improvements brought to the scattering model affect model performance less than the improvements brought to FRTMs. Scattering depends on the optical properties of leaves, wood and soil, and the considered radiation wavelength. Radiation scattering is especially low in PAR and in ultraviolet radiation $(400-700 \mathrm{~nm})$ (Parker, 1997; Sinoquet et al., 1993). The reflectivity of a green leaf is approximately 0.2 and this value is even lower for an entire crown because of multiple scattering (Brunner, 1998; Landsberg and Sands, 2010). Moreover, scattering models require large numbers of parameters (Kuusk, 1993) and much computation time (Rey et al., 2008; Ross, 1981). Consequently, the use of a scattering model has often been neglected in forestry assuming that leaves and ground behave like black bodies within the PAR waveband (i.e. showing no transmittance and no reflectance)
(Sinoquet et al., 1993) even though it might improve the precision of the prediction of transmitted irradiance (Mariscal et al., 2004).

Given the variety of applications and newly developed modeling approaches of FRTMs, it would be of great help for modelers to know which approach enables them to predict the variable of their interest with a given precision, a low bias, and accepted calibration efforts. Moreover, having insight of the expected precision and bias associated with a modeling approach is also precious information in assessing whether it would fulfill the study objectives. Similarly, knowing beforehand to which parameters the models are most sensitive and the order of magnitude of parameter values can speed up and improve the calibration work. This would allow for effort to be invested in estimating precisely the sensitive parameters and using simplification assumptions for the less sensitive parameters.

Unfortunately, as far as forests are concerned, the performance analyses and the sensitivity analysis of different modeling approaches have been reported separately and for very restricted sets of canopy conditions and study objectives. The information is therefore scattered and no general guideline has been formulated to help forest modelers to choose which modeling approach suits best his needs.

In this paper, we synthesized both the performance analyzes and the sensitivity analyses of radiation transfer models through forest canopies. After classifying these approaches, we attempted to quantify the expected uncertainty and appraise the calibration efforts associated with most combinations of modeling approaches and model applications. We provided order of magnitude estimates for the main calibration parameters and attempted to report how sensitive models are to these parameter changes. Finally, we attempted to identify the modeling approaches that best suit to the different applications of FRTMs.

\section{Classification of FRTMs}

We identified four families of FRTMs that combine three approaches to describe the geometry of forest canopy and two approaches to compute the proportion of incident radiation intercepted by the canopy (Table 1). Below, we briefly describe and discuss each of these approaches; details can be found in previous articles (Brunner, 1998; Cescatti, 1997; Lieffers et al., 1999; Sinoquet et al., 1993).

\subsection{Canopy geometry}

\subsubsection{One-dimensional canopy}

The simplest approach for modeling the geometry of a forest canopy is to assimilate it to a single horizontal layer of vegetation without individualizing crowns or trees (one-dimensional (1D) model). Due to its simplicity, this approach is particularly appropriate modeling a homogeneous canopy such as the one of pure even-aged stands. This approach is more challenging with heterogeneous stands as additional assumptions and 


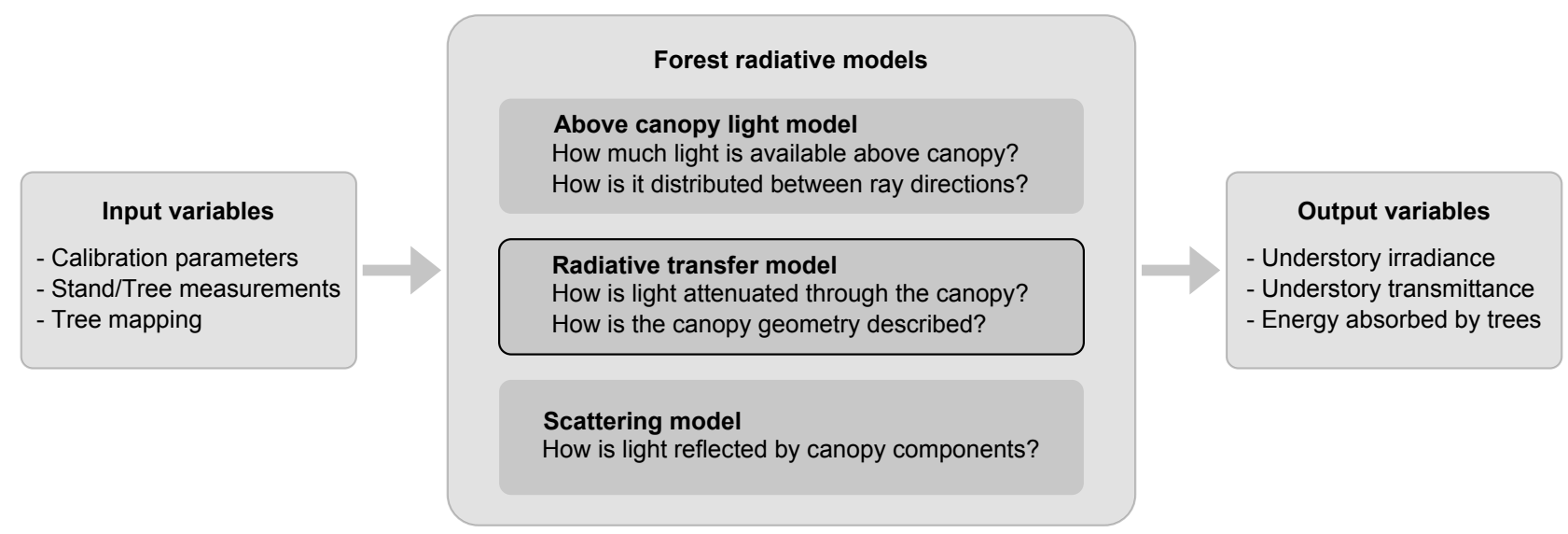

Figure 1. Chart flow of forest radiative model indicating the three submodels.

Table 1. Classification of the modeling approaches used to model forest radiative transfer.

\begin{tabular}{|c|c|c|c|c|}
\hline FRTM family & Abbreviation & Canopy & Transmittance & Main objectives \\
\hline 1D model & $1 \mathrm{D}$ & $\begin{array}{l}\text { Stand canopy is composed of } \\
\text { one or several horizontal lay- } \\
\text { ers }\end{array}$ & Turbid medium & $\begin{array}{l}\text { Ecophysiological processes } \\
\text { at stand level }\end{array}$ \\
\hline $\begin{array}{l}\text { 3D crown model with } \\
\text { turbid medium }\end{array}$ & 3D-TM & $\begin{array}{l}\text { Tree crowns are composed } \\
\text { of one or a set of geometric } \\
\text { shapes }\end{array}$ & Turbid medium & $\begin{array}{l}\text { Forest growth and yield; Dy- } \\
\text { namics of stand structure }\end{array}$ \\
\hline $\begin{array}{l}\text { 3D crown model with } \\
\text { porous envelope }\end{array}$ & 3D-PE & $\begin{array}{l}\text { Tree crowns are composed } \\
\text { of one or a set of geometric } \\
\text { shapes }\end{array}$ & Porous envelope & $\begin{array}{l}\text { Forest growth and yield; Dy- } \\
\text { namics of stand structure }\end{array}$ \\
\hline 3D surface model & $3 \mathrm{D}-\mathrm{S}$ & $\begin{array}{l}\text { Trees are composed of sur- } \\
\text { faces representing the leaves, } \\
\text { branches and stems }\end{array}$ & $\begin{array}{l}\text { Porous envelope or ra- } \\
\text { diative transfer theory }\end{array}$ & $\begin{array}{l}\text { Tree architecture; ecophysio- } \\
\text { logical processes at tree level }\end{array}$ \\
\hline
\end{tabular}

Note : FRTM, forest radiative transfer model; 1D, one-dimensional; 3D, three-dimensional.

parameters are required (Duursma and Mäkelä, 2007; Kim et al., 2011) to offset the simplicity of the canopy description.

The model developed by Kim et al. (2011) illustrates well this approach. This model requires calibrating for each species, $j$, three extinction coefficients (corresponding to the distribution of inclination angles of leaves, branches and stems), the one-sided area of leaves, branches and stems, the horizontal crown projection and tree density. Some of these parameters might also depend on ray directions (i.e. ray zenith angle $(\eta)$ and ray azimuthal angle $(\gamma)$ ). Furthermore, the model assumes that branches and stems are randomly distributed, whereas the distribution of leaves depends on a clumping factor. Simpler approaches with fewer variables and empirical parameters have been developed. For instance, Duursma and Mäkelä (2007) used a 1D model with four variables (leaf area, crown surface area, number of trees and extinction coefficient) and one empirical parameter.

Another solution to modeling the distribution of leaves consist of subdividing the canopy into several homogeneous regions, e.g. horizontal layers, on the condition that the vertical structure of the stand can be approximated. Such as multilayer models can then predict understory light at different canopy heights.

Moreover, due to the main hypothesis of considering continuous layer(s) of vegetation with the same properties for the whole forest stand we did not expect the 1D model to predict accurately the spatial variation of irradiance beneath the canopy. The 1D model is rather utilized to predict the temporal variation of irradiance (Govind et al., 2013; Kim et al., 2011).

\subsubsection{D crown models}

Alternatively, 3D crown models (see 3D-TM and 3D-PE in table 1) assimilate forest stands into a set of spacialized geometric shapes representing tree crowns and, optionally, trunks. Many different geometric shapes have been used (Table 2). The simplest are quadric surfaces such as the cylinder, cone, ellipsoid or paraboloid. Such shapes have the advantages of 
requiring few parameters and allowing analytical computation of the interceptions between light rays and crowns. In contrast, nonquadric shapes (e.g. combinations of degenerated surfaces) fit well to real crowns but require numerous parameters and more complex numerical computations.

Many refinements to 3D crown models have been described in the literature, and have been notably reviewed by Brunner (1998). For example, crowns can be divided into different parts characterized by different leaf densities. A combination of several shapes can be used to distinguish an illuminated surface (e.g. upper half ellipsoid) from a shaded surface. Similarly, a special foliage envelope can be constructed within a crown, for example with branches that have developed during the last 5 years. Missing parameters are often estimated using allometric relationships to diameter at breast height (Da Silva et al., 2012) or relationships to species shade tolerance (Beaudet et al., 2011; Canham et al., 1999). Algorithms of crown reconstruction according to neighbor competition are also available (Piboule et al., 2005).

In brief, the recent improvements brought to $3 \mathrm{D}$ crown models attempted to model crown geometry as realistically as possible taking into account that trees often lean and crown shapes often deviate from simple geometric forms. Hence, they required adjusting a greater set of parameters, and some of them can hardly be measured in the field and must be estimated with additional models.

Moreover, on one hand, the 3D crown model requires determining the position and crown dimensions of every tree whereas the 1D model requires only estimating stand features. On the other hand, measuring crown dimensions is easier and more commonly performed than estimating foliage aggregation within the stand canopy. Additionally, taking explicitly into account stand heterogeneity, 3D crown models can predict the spatial variability of transmitted light.

\subsubsection{D surface models}

3D surface models (3D-S) represent leaves, branches and stems as realistically as possible with surfaces or shells. This leads to very detailed 3D mock-ups (Fig. 2) with components that are usually assimilated into opaque envelopes (see section 2.2.2). Due to the number of input parameters for these models, they are used to represent single trees (Sinoquet et al., 2001), orchard trees (Da Silva et al., 2008), or agroforestry systems (Dauzat and Eroy, 1997; Leroy et al., 2009) rather than entire forest stands. Homogeneous forest stands could be obtained by replicating one or a few model trees (Fig. 2), but it seems unlikely to be able to gather all the necessary information to model every single tree of one heterogeneous stand. Hence, we believe that the use of 3D-S models in forestry is nowadays limited to applications involving tree architecture and ecophysiological processes.

\subsection{Radiation attenuation}

\subsubsection{Turbid medium}

The first approach to computing the fraction of transmitted light through the canopy $(\tau)$ consists of using an analogy

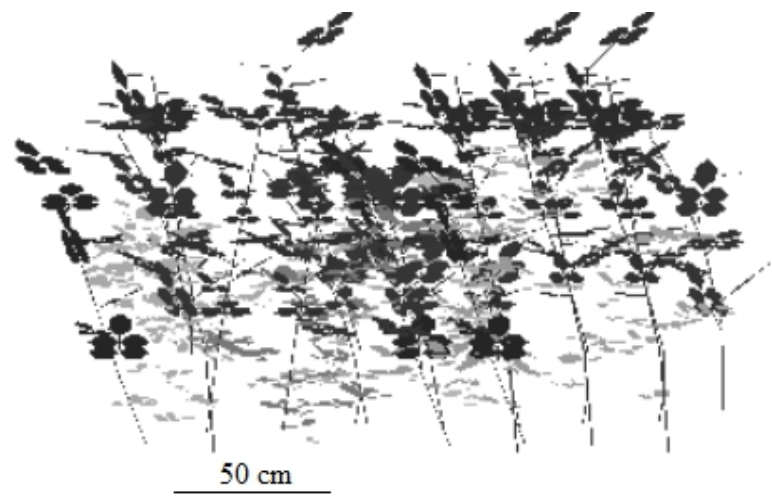

Figure 2. Overview of FRTM models according to their end-uses. Most FRTMs included in stand dynamic models are 3D crown models, whereas most FRTMs used for ecophysiological studies are 1D models. LAD, leaf area density; $k$, extinction coefficient; $\Omega$, clumping factor; $p$, crown openness; 1D, one dimensional; 3D, three-dimensional.

to Beer's law. Beer's law describes the attenuation of a monochromatic ray within a turbid medium, i.e. a medium made up of small elements randomly scattered and presenting a homogeneous transparency (Brunner, 1998). Canopy is assimilated to such a turbid medium and $\tau$ is computed as a function of the density and the spatial distribution of canopy elements such as leaves and branches (Lieffers et al., 1999; Sinoquet et al., 1993). The turbid medium analogy can be applied to an entire stand canopy, a horizontal layer within a stand canopy, a crown envelope, or even a part of a crown. This approach relies on the assumption that canopies have the properties of such a homogeneous medium. Nevertheless, stand canopies or tree crowns are made up of various size elements often aggregated within crowns and vertices. Therefore, several correction coefficients have been developed to adapt Beer's law to canopies. Briefly, the probability of beam interception $(1-\tau)$ by canopy elements is a function of the canopy element density (leaf area density, LAD $\left(\mathrm{m}^{2} \mathrm{~m}^{-3}\right)$, the path length of a ray through the canopy $(l)$, the extinction coefficient $(k)$ and the clumping factor $(\Omega)$. $k$ and $\Omega$ depend on canopy element inclination and spatial distribution, respectively (eq. 1). $\Omega$ describes the aggregative pattern of branches and leaves within the canopy. Many different mathematical expressions have been used to adapt the Beer's law models to $1 \mathrm{D}$ and $3 \mathrm{D}$ models, but all somehow include these three parameters. Equation 1 is the expression commonly used to compute $\tau$ with 3D crown models.

$$
\tau(\eta, \gamma)=\exp (-k \cdot \Omega \cdot L A D \cdot l(\eta, \gamma))
$$

The advantage of the turbid medium approach lies mainly in its mechanistic formulation and the use of the leaf area because leaf area is a key variable in forest ecology (e.g. used to model photosynthesis and transpiration). However, this 
Table 2. Examples of 3D-geometric crown models used in FRTMs. Most authors used relatively simple quadric shapes whereas few tested more complicated shapes.

\begin{tabular}{lll}
\hline & Quadric shapes & $\begin{array}{l}\text { Combination of de- } \\
\text { generated shapes }\end{array}$ \\
$\begin{array}{l}\text { No. of param- } \\
\text { eters } \\
\text { References }\end{array}$ & $\begin{array}{l}\text { Koop and Sterck (1994); Bartelink } \\
\text { (1998b); Beaudet et al. (2002); Cour- } \\
\text { baud et al. (2003); Stadt and Lief- } \\
\text { fers (2000); Mariscal et al. (2004); } \\
\text { Beaudet et al. (2011); Canham et al. } \\
\text { (1994); Pinno et al. (2001); Pukkala } \\
\text { et al. (1993); Paquette et al. (2008) }\end{array}$ & $\begin{array}{l}\text { Cescatti (1997); } \\
\text { Brunner (1998); Pi- } \\
\text { boule (2001); Groot } \\
\text { (2004); Piboule et al. } \\
\text { (2005); Da Silva et al. } \\
\text { (2012) }\end{array}$ \\
\hline
\end{tabular}

approach often requires empirical estimation of $k$ and $\Omega$, and leaf area measurements that remain both difficult to obtain and imprecise (Breda, 2003). Moreover, this is the only approach that applies if the canopy geometry is described with a 1D model.

\subsubsection{Porous envelope}

The second approach, called porous envelope, assumes that crowns or parts of crown are envelopes with one empirically estimated parameter, namely the crown openness ( $p$, eq. 2 ), which is the probability of a ray being intercepted by the foliage. This approach assumes that leaves do not transmit or reflect light (like black bodies). This assumption can be more easily verified for coniferous species with dark foliage than for broadleaved species with lighter foliage (Williams, 1991). Therefore, some authors have adjusted equation 2 for the different radiation wavelength ranges (Goudriaan, 1977). The variable $p$ is independent of ray direction and path length (l) (Boivin et al., 2011; Canham et al., 1994; Da Silva et al., 2008; Groot, 2004) and is also defined as the fraction of sky visible through a crown (Canham et al., 1999).

$$
\tau(\eta, \gamma)=p
$$

In comparison with the previous approach, this one is less mechanistic but requires fewer parameters (usually one per species) and is therefore easier to calibrate. On the other hand, this approach describes the attenuation of radiation through crowns and not through stand canopy. Therefore it cannot be utilized with a 1D model.

Other simplifications of these submodels have also been proposed. For example, Koop and Sterck (1994) used an opaque crown model $(p=0)$. Other authors have simplified the turbid medium submodel by removing $l, \Omega$ and LAD from equation 1 . In this way, they obtained a "hit model" with a formulation (eq. 3) very close to equation 2 with one empirical parameter, which they called the extinction coefficient (Boivin et al., 2011; Canham et al., 1994; Koop and Sterck, 1994).

$$
\tau(\eta, \gamma)=\exp (-k)
$$

\section{Input variables}

Different input data and calibration parameters are required depending on the chosen modeling approach. These parameters are measured, estimated or adjusted by model inversion. Below, we give an overview of model requirements in terms of input data and summarize their definitions and estimation methods.

\subsection{Stand and tree measurements}

Among all FRTM inputs, stand and tree measurements probably require the most workforce and consequently represent one of the major cost in developing FRTM. As far as stand and tree measurements are concerned 1D models obviously require less field data than $3 \mathrm{D}$ models and, hence, require less field work. 1D models depend mainly on foliage density and distribution (see sections 3.2, 3.3, 3.4 which does not require carrying out intensive tree inventory. In most cases, measuring stand density is enough. However, 3D models often rely on an intensive inventory and mapping of every tree within a plot. For 3D crown models, at least three parameters are generally needed for each tree: height, crown base height and crown radius (Table 2). Additional measurements such as crown radii measured in different directions, height of maximum 
crown extension, and shape coefficients might be required to construct more complex shapes. For 3D surface models, a geometric description of leaves, branches, and stems can be obtained by vectorization (Fournier et al., 1996), digitalization (Moulia and Sinoquet, 1993), or simulations of plant morphology (Leroy et al., 2009).

\subsection{Density of canopy elements}

The stand leaf area index (LAI, $\mathrm{m}^{2} \mathrm{~m}^{-2}$ ) is the total one-sided foliage area per unit of soil surface. LAI is the primary descriptor of plant canopy and a key variable in studying plant physiological processes (e.g. photosynthesis, transpiration). In the field, stand LAI is directly assessed with litterfall traps or vertical line intercept sampling coupled with measures of leaf inclination. LAI can also be estimated indirectly with optical methods, mainly using hemispherical photographs or LAI-2000 devices (LI-COR Biosciences, Lincoln, Nebraska, USA) (Jonckheere et al., 2004; Weiss et al., 2004). These last two methods measure the gap fraction (i.e. the fraction of sky visible from the measuring point) and use the turbid medium analogy to infer leaf area. Optical approaches therefore rely on the same assumptions and parameters as eq. 1 . Moreover, such methods give estimates of the effective LAI, which includes the area of branches and trunks (Jonckheere et al., 2004). Additionally, reference values and allometric relationships are available in the literature for the most common species.

Measurements of leaf area for individual trees are very difficult to replicate for every tree in a stand, even though individual LAI values are necessary with 3D-TM. Therefore, tree leaf area is often estimated for each species by model inversion (Courbaud et al., 2003) or by measuring LAD on a sample of trees (Stadt and Lieffers, 2000). Nevertheless, as indicated by Nock et al. (2008), intracrown leaf area decreases with tree age by up to $40 \%$. It might therefore be preferred to use allometric relationships with, for example, tree diameter (Bartelink, 1998a; Gersonde et al., 2004), tree height (Essery et al., 2008), sapwood area (Gersonde et al., 2004), or tapering equations (Kim et al., 2011), or by the inversion of a turbid medium model.

Moreover, LAI is often used with a $1 \mathrm{D}$ model, whereas LAD is usually preferred with $3 \mathrm{D}$ crown models. LAD corresponds to the one-sided leaf area divided by the canopy element modeled volume (Gersonde et al., 2004). The reported values of LAD used with 3D-TM models range from 0.3 to $6 \mathrm{~m}^{2} \mathrm{~m}^{-3}$ (Table 3 ). This variation is therefore substantial even for the same species. This encourages improving and harmonizing the methods used to estimate LAD.

Some simplifications used to estimate foliage density might appear rough to some readers but they reflect well the difficulty of measuring it; due to tree size and heterogeneity, its measure in forests is much more challenging than in crops or orchards.
Table 3. Reported values of leaf area density (LAD, $\mathrm{m}^{2} \mathrm{~m}^{-2}$ ) used in 3D crown models with the turbid medium analogy (3D-TM)

\begin{tabular}{|c|c|c|}
\hline species & LAD & Source \\
\hline not specified & 1.86 & $\begin{array}{l}\text { (Kuuluvainen and Pukkala, } \\
\text { 1991) }\end{array}$ \\
\hline \multicolumn{3}{|l|}{ Coniferous } \\
\hline Pinus ponderosa & $0.03-0.36$ & Law et al. (2001) \\
\hline Tsuga heterophylla & 0.38 & Mariscal et al. (2004) \\
\hline Pseudotsuga menziesii & $\begin{array}{l}0.38 ; \quad 0.96 \\
2.74 ; 6.00\end{array}$ & $\begin{array}{l}\text { Brunner (1998); Gersonde } \\
\text { et al. (2004); Mariscal et al. } \\
\text { (2004); Webb and Ungs } \\
\text { (1993) }\end{array}$ \\
\hline Picea abies & 0.4 & Courbaud et al. (2003) \\
\hline Pinus contorta & 1.39 & Stadt and Lieffers (2000) \\
\hline Picea glauca & $1.80-1.88$ & Stadt and Lieffers (2000) \\
\hline Abies balsamea & 1.98 & Stadt and Lieffers (2000) \\
\hline not specified & 2 & Sprugel et al. (2009) \\
\hline Pinus ponderosa & 2.01 & Gersonde et al. (2004) \\
\hline Pinus lambertiana & 2.56 & Gersonde et al. (2004) \\
\hline Abies concolor & 3.36 & Gersonde et al. (2004) \\
\hline Calocedrus sp. & 4.51 & Gersonde et al. (2004) \\
\hline \multicolumn{3}{|l|}{ Broadleaves } \\
\hline Populus balsamifera & 0.3 & Stadt and Lieffers (2000) \\
\hline Populus tremuloides & 0.44 & Stadt and Lieffers (2000) \\
\hline Fagus sylvatica & 0.66 & Piboule (2001) \\
\hline not specified & $0.5-1$ & Sprugel et al. (2009) \\
\hline Betula pendula & 0.79 & Piboule (2001) \\
\hline Betula papyrifera & 0.8 & Stadt and Lieffers (2000) \\
\hline Quercus kelloggii & 1.32 & Gersonde et al. (2004) \\
\hline
\end{tabular}

\subsection{Extinction coefficients}

The extinction coefficient, $k$, is a parameter of eq. 1 and is therefore used whenever the radiation attenuation submodel corresponds to the turbid medium approach. It is usually a species-specific constant but it can also be computed as a function (the "G-function"), which depends mainly on the orientation and inclination of leaves as well as on the ray zenith angle $(\eta)$. The parameter $k$ has been estimated by measuring leaf inclination and projected leaf areas (Campbell and Norman, 1998; Kim et al., 2011), computed with theoretical functions of leaf distribution function (Govind et al., 2013) or deduced from the relationship between transmittance and LAI measured at different canopy heights (Landsberg and Sands, 2010).

With 3D crown models, most authors assume that leaf inclination follows a theoretical distribution that is either spherical with $k=0.5$ (Brunner, 1998; Cescatti, 1997; Courbaud et al., 2003; Piboule, 2001) or ellipsoidal (Stadt and Lieffers, 2000). With 1D models, authors have sometimes preferred using empirical estimates of $k$ ranging between 0.2 and 0.6 (Aubin et al., 2000; Bartelink, 1998b; Jarvis and Leverenz, 1983; Pierce and Running, 1988).

\subsection{Clumping factors}

The assumption of the random spatial distribution of canopy elements is rarely satisfied (Da Silva et al., 2008; Sinoquet et al., 2005), especially with 1D models, because the spatial distribution of trees, but also of canopy elements within trees, is rarely completely random. Moreover, leaves are gathered around branches, and branches are grouped around stems 
and sometimes grouped in vertices. Whenever the spatial distribution of canopy elements is not known or approximated (e.g. stand map, tree architecture), a clumping factor, $\Omega$, is required in eq. 1. Indeed, a canopy with regularly spaced elements $(\Omega \geq 1)$ transmits less light than a canopy with randomly scattered elements $(\Omega=1)$. By contrast, a canopy with aggregated elements transmits much more light $(\Omega \leq 1)$ (Niinemets, 2010).

Like the extinction coefficient, the clumping factor is rarely measured because measuring it for adult trees is very labor intensive. Consequently, it is often assumed to be constant. There is a lack of reference values for the clumping factor and this measurement is therefore often estimated by model inversion.

With 1D models, Chen et al. (1999) used values of the clumping factor of 0.5 and 0.7 for conifers and broadleaves, respectively. These values were computed by measuring the canopy gap size distribution (Chen et al., 1997). Kim et al. (2011) estimated the clumping factor by harvesting pine shoots. They observed that the clumping factor varied between 0.2 (upper part of the canopy) and 0.9 (lower part of the canopy), while other studies reported values for long-needled species ranging between 0.4 and 0.9 (Thérézien et al., 2007). In addition, some studies have demonstrated a variation in the clumping factor with stand density, crown diameter and ray zenith angle (Govind et al., 2013; Wang and Jarvis, 1990). Because tree aggregation is already taken into account with $3 \mathrm{D}$ crown models, the clumping factor in these models is usually assumed to be 1 .

\subsection{Crown openness}

Crown openness $(p)$ can be assessed by photographing isolated crowns (Astrup and Larson, 2006; Beaudet et al., 2011, 2002; Canham et al., 1999, 1994; Da Silva et al., 2012; Paquette et al., 2008) or by model inversion (Groot, 2004). Photographs can be taken using either fish-eye lens (Astrup and Larson, 2006; Beaudet et al., 2011, 2002; Canham et al., 1999, 1994) or classic lens (Da Silva et al., 2012). In comparison with fish-eye lenses, classic lenses allow smaller isolated parts of the crown to be photographed. Therefore, the use of a classic lens makes it easier to obtain a correct exposure even when sky conditions are not overcast. Subject crowns need to be isolated with no overlapping neighboring crowns. Photographs are processed in order to compute the proportion of sky pixels (i.e. the crown openness). Boivin et al. (2011) presented an algorithm that identifies the crown extent and argued that crown delimitation has a strong influence on porosity estimates. Canham et al. (1994) observed no variation in $\mathrm{p}$ with zenith angle and tree dimensions. However, Astrup and Larson (2006) observed that $p$ varied, to a limited extent, in spruce stands with tree diameter and by regions.

Crown openness $(p)$ and LAD are two related parameters. Indeed, Da Silva et al. (2012) bridged the two approaches proposing a method to compute $k$ LAD from $p$. The two approaches of modeling light attenuation through canopy, namely the turbid medium and porous envelope approaches, can therefore be calibrated with field measurements of $p$. Even though it applied only to isolated trees, the measurement of $p$ is more convenient and less laborious than the measurement of LAD. Moreover, the reported variability of $p$ seems lower than that of LAD with values ranging from 0.03 to 0.29 and, an average around 0.1 (table 4 ).

Table 4. Reported estimates of crown openness used with $3 \mathrm{D}$ crown models (3D-PE).

\begin{tabular}{|c|c|c|}
\hline Species & Estimates & References \\
\hline \multicolumn{3}{|l|}{ Broadleaves } \\
\hline Fagus sylvatica & 0.048 & Da Silva et al. (2012) \\
\hline Fagus grandifolia & 0.05 & Beaudet et al. (2002) \\
\hline Malus sylvestris & 0.052 & Da Silva et al. (2012) \\
\hline Quercus petreae & $0.043-0.073$ & Da Silva et al. (2012) \\
\hline Betula papyrifera & 0.058 & Canham et al. (1999) \\
\hline Populus tremula & 0.082 & Da Silva et al. (2012) \\
\hline Sorbus torminalis & $0.049-0.120$ & Da Silva et al. (2012) \\
\hline Betula alleghaniensis & 0.097 & (Beaudet et al., 2002) \\
\hline Betula papyrifera & 0.101 & Beaudet et al. (2011) \\
\hline Acer saccharum & 0.108 & Beaudet et al. (2002) \\
\hline Populus balsamifera & 0.14 & Canham et al. (1999) \\
\hline Populus tremuloides & $0.163 ; 0.183$ & $\begin{array}{l}\text { Astrup and Larson (2006); } \\
\text { Beaudet et al. (2011) }\end{array}$ \\
\hline Betula alleghaniensis & 0.206 & Canham et al. (1999) \\
\hline Populus hybrid & 0.3 & Paquette et al. (2008) \\
\hline \multicolumn{3}{|l|}{ Coniferous } \\
\hline Abies alba & 0.034 & Da Silva et al. (2012) \\
\hline Abies amabilis & 0.06 & Canham et al. (1999) \\
\hline Tsuga heterophylla & 0.08 & Canham et al. (1999) \\
\hline Pinus sylvestris & $0.065-0.110$ & Da Silva et al. (2012) \\
\hline Abies lasiocarpa & 0.09 & Canham et al. (1999) \\
\hline Thuja plicata & 0.09 & Canham et al. (1999) \\
\hline Picea glauca & $\begin{array}{l}0.109 ; \quad 0.110 \\
0.130\end{array}$ & $\begin{array}{l}\text { Beaudet et al. (2011); Canham } \\
\text { et al. (1999); Astrup and Lar- } \\
\text { son (2006) }\end{array}$ \\
\hline Abies balsamea & 0.111 & Beaudet et al. (2011) \\
\hline Pinus banksiana & 0.124 & Beaudet et al. (2011) \\
\hline Pinus contorta & 0.135 & Canham et al. (1999) \\
\hline Thuja occidentalis & 0.144 & Beaudet et al. (2011) \\
\hline Picea mariana & $0.070-0.290$ & Groot (2004) \\
\hline
\end{tabular}

\section{Model applications and output variables}

Because FRTMs are most often used in order to model forest ecosystem functioning, they are usually coupled with many other models describing, for example, photosynthesis, transpiration, tree growth, timber production, carbon sequestration, nutrient uptake, or hydrological balance. The applications of FRTMs are therefore numerous but they can be seen, for the sake of simplicity, as either ecophysiological applications or stand-dynamic applications. Ecophysiological studies uses FRTMs coupled mainly with process-based models that embody our current knowledge of the functioning of forest ecosystem. Such holistic models include, for instance, equations describing the fluxes of carbon, water, and energy between soil, vegetation, and atmosphere (Govind et al., 2013). Such studies aim at improving our understanding of forest ecosystem functioning, exploring forest productivity as a function of resource availability, or at exploring how variation of forest structure and composition can affect ecosystem 
functioning and dynamics. Forest productivity, standing forest biomass, nutrient uptake or hydrological balance as examples can then be predicted for particular environmental conditions and for forecasted modifications of these. Within this context, FRTMs are necessary to predict the irradiance $\left(\mathrm{W} \mathrm{m}^{-2}\right)$ and (or) the spectrum of the intercepted radiations at various scales, from a global scale to a leaf scale.

The second group of FRTMs studies preferentially use empirical models. They bring less concerns on ecosystem functioning and focus rather on the dendrometric data of interest for forest managers. They aim mainly at predicting the natural evolution of stand structure and composition and (or) predicting the effect of overstory management on understory light conditions (Beaudet et al., 2011; Beaudet and Messier, 2002; Courbaud et al., 2001; Sprugel et al., 2009). Also, their objective is the best possible prediction of forest growth and the outcome of intra and inter specific competition. Such models can more precisely predict forest dynamics but, contrary to process-based models, their uses are more strictly limited to the conditions from where they were derived. On the other hand, using empirical models of tree growth allows focusing on light availability without calibrating and programming all of the physiological processes in relation to tree growth. This simplification has often been justified whenever light can be considered as the first limiting factor of vegetation growth (e.g. tree regeneration under closed canopy). Within this context, FRTMs are needed to predict tree irradiance, i.e. the amount of energy absorbed by trees (Courbaud et al., 2003), the proportion of radiations transmitted (transmittance) to the regeneration (Beaudet et al., 2011; Beaudet and Messier, 2002; Lieffers et al., 1999; Pacala et al., 1996; Sprugel et al., 2009), the amount of light available for understory biodiversity (Balandier et al., 2006; Barbier et al., 2008), or the vertical gradient of transmittance within the canopy (Gersonde et al., 2004; Mariscal et al., 2004). They are used mostly at plot or stand scales (from $100 \mathrm{~m}^{2}$ to 1 ha) with a time step of 1 year.

\section{Model evaluation}

Most authors evaluate their model by comparing estimates and measurements of irradiance or transmittance taking into account either total incident light or only the incident diffuse light (e.g. indirect site factor). Light measurements are performed usually with hemispherical photographs and less frequently with light sensors. Hemispherical photography is an indirect method with an associated level of error that can occasionally be substantial. Moreover, this form of photography uses a radiative model to assess irradiance and transmittance. Using hemispherical photography to evaluate FRTMs therefore leads to a comparative evaluation of one radiative model with another. In addition, FRTMs have been evaluated for their performance in predicting irradiance or transmittance at the ground level, above the understory vegetation, or, occasionally, at different heights within a tree canopy (Kim et al., 2011; Mariscal et al., 2004). In the present study, we compiled published information on the performance of different models, i.e. their ability to predict the measurements with low levels of error and bias. We considered all published light models that were used to predict radiative transfer through forest canopies and for which the performance was evaluated. In Table 5, we summarized the reported performance of these models. Unfortunately, authors have used different approaches to assess model performance, making comparison difficult. They often adjusted linear models between estimates and observations and hence assessed model precision using the coefficient of determination $\left(\mathrm{R}^{2}\right)$ or the root mean square error (RMSE). Model bias was usually examined graphically but some authors have used the coefficients of the linear regression between measurements and estimates. Moreover, even similar indicators (e.g. $\mathrm{R}^{2}$ ) could not be compared without caution. While most authors compared estimates and measurements carried out at different periods (Govind et al., 2013; Kim et al., 2011) or at different positions within plots, others compared average estimates and measurements between sites of areas ranging from 900 to $1600 \mathrm{~m}^{2}$ (Stadt and Lieffers, 2000) or the average gap profile (Mariscal et al., 2004). In addition, others compared the statistical distribution of transmittance estimates and measurements (Da Silva et al., 2012). Finally, the numbers of plots and sites were also far from constant between model evaluations.

Most authors found good agreement between measurements and estimates when averaged at the stand scale (Mariscal et al., 2004; Stadt and Lieffers, 2000). In other words, most models predicted transmitted light poorly at a particular location (at sensor scale) beneath the canopy but rather well at the stand scale. After a good calibration, the relationship between measurements and predictions was found to be generally close to a 1:1 relationship and hence without unacceptable bias in the point-to-point comparison. Nevertheless, large deviations were often observed between estimates and measurements of transmittance (often up to 20\%) even for low light levels. Many authors argued that point-to-point variations beneath the forest canopy are rarely predicted with accuracy because minor errors in tree position and crown dimensions can lead to dramatic changes in simulations of transmittance (Da Silva et al., 2012; Fournier et al., 1996; Groot, 2004; Mariscal et al., 2004). On the other hand, site averages were better predicted (Da Silva et al., 2012; Mariscal et al., 2004; Pinno et al., 2001; Stadt and Lieffers, 2000). Similarly, model performance increased with the length of the simulated period (e.g. 1 year versus $1 \mathrm{~h}$ ) especially when direct radiations were taken into account (Brunner, 1998; Groot, 2004).

All of the different modeling approaches were reported to perform well but few authors compared the different approaches. Kim et al. (2011) found that their 1D model performed as well as 3D crown models when they took into account the aggregation of trees, leaves and branches with appropriate clumping factors. Similarly, Balandier et al. (Year) obtained very similar results with a 1D model and a 3D surface model in a mixed understory of European beech (Fagus sylvatica L.) and European red raspberry (Rubus idaeus L.) (Fig. 
2). 3D-TM (e.g. MIXLIGHT) and 3D-PE (e.g. SORTIE-ND) appeared to exhibit a very similar performance, although Canham et al. (1994) found that a hit model (i.e. 3D-PE with $p=0$ ) performed better than a 3D-TM. These authors interpreted this result as an effect of foliage scattering at the crown periphery, especially for shade intolerant species. Boivin et al. (2011) used a 3D-PE model and obtained good results in plantations of hybrid poplars, with $\mathrm{p}=0$, whereas in the same study, this parameter value provided the worst results in young boreal mixedwoods.

Nevertheless, we noted that the relative performance depended on the modeled description of canopy geometry (Table 5). The highest levels of model performance were obtained with very detailed 3D-TM models (Brunner, 1998; Cescatti, 1997). These models used asymmetric crowns described by a combination of nonquadric shapes and modeled the radiative transfer using a turbid medium approach with $k=0.5$ and $\Omega=1$. In addition, Brunner (1998) restricted the foliage to shells within the crown but acknowledged that simplification of his canopy model would have altered the results only slightly. Moreover, he restricted his sample to a single even-aged coniferous stand with a large gradient of transmittance, which made model calibration and validation easier. The model of Cescatti (1997) differed from the model developed by Brunner (1998) in its inclusion of an algorithm for scattering processes.

Additionally, model performance appears to be related to forest structure (Table 5). Model performance decreases from even-aged young stands to multi-layered and mixed mature stands. This observation is well supported by pairs of publications in which the same model has been applied to different forest structures. For instance, the model FOREST was shown to provide excellent results in an 80-year-old spruce stand in Finland (Cescatti, 1997), whereas it provided only moderate results in a multilayered forest of ponderosa pine in Oregon (Law et al., 2001). Similarly, using the model tRAYci, Brunner (1998) successfully predicted the spatial distribution of the transmittance under a 20-year-old stand of Douglas fir, whereas Gersonde et al. (2004) obtained a lower model performance with a mixed conifer forest. MIXLIGHT successfully predicted point-to-point variation in transmittance under aspen-dominated stands aged from 1 to 30 years (Pinno et al., 2001), but it was less successful in predicting the average values of transmittance in various even-aged boreal stands aged from 69 to 159 years (Stadt and Lieffers, 2000). Finally, Boivin et al. (2011) observed the same trend between young boreal forests and hybrid poplar plantations, and Koop and Sterck (1994) found that including measurement points located close to small trees reduced the $\mathrm{R}^{2}$ from 0.94 to 0.77 .

\section{Model sensitivity}

We attempted to identify which model parameter was reported to have strong effect on FRTM performance. As many studies actually lack a common referenced method, we classified the studied parameters into three qualitative levels according to their impact on FRTM predictions: high, medium, and low.
This classification was based mainly on the discussion by the respective authors as part of their studies. For example, given the results of Gersonde et al. (2004), LAD and crown radius were classified respectively in the low and high categories. Indeed, these authors observed that replacing individual LAD with a species average did not reduce the model fit. On the other hand, in the same study, the same simplification applied to the crown radius reduced very significantly model fit $\left(\mathrm{R}^{2}\right.$ was reduced from about $75 \%$ to $65 \%$ ). Next, we counted the number of publications of each combination of parameter and sensitivity level (Table 6). Unfortunately, not all of the parameters had been tested in similar conditions or with the same model, as far as forest stands are considered. We therefore recognize that our analysis is incomplete, but underlines well the common trends between different studies.

As a general rule, we found that models appeared more sensitive to the parameters describing between-crown gaps than to those describing within-crown gaps or within-crown architecture. In most studies, a larger amount of light was transmitted between rather than through the crowns. Indeed, as shown by the estimates of crown openness in Table 4 , only $5 \%$ to $30 \%$ of incident light passes through the crown. The parameters describing the between-crown gap geometry were therefore of primary importance, justifying the use of a hit model (3D-PE with $p=0$ ) for certain types of stands. Nevertheless, we found that authors often noted that FRTMs were more sensitive to other parameters such as tree clumping or leaf area in dense stands (Boivin et al., 2011; Wang and Jarvis, 1990).

Stand density and stand edges are not model calibration parameters but they greatly affect transmitted light estimates (Bartelink, 1998b; Piboule, 2001). Moreover, it is even possible that stand density modifies model sensitivity to the inner-crown parameters. In dense and homogeneous stands, most rays were found to intercept crowns, whereas in lowdensity stands, the majority of transmitted radiation comes from canopy gaps. Models are thus found to be less sensitive to inner-crown parameters with low-density stands.

\subsection{D model}

The main source of variability of 1D models was found in the areas covered by crowns (Duursma and Mäkelä, 2007) and leaves (Bertin et al., 2011; Duursma and Mäkelä, 2007; Kim et al., 2011). These parameters appeared to be highly influential in all the sensitivity analyses that studied their effects. These estimates were further corrected with clumping factors that took into account the aggregation of stems, branches, and (or) leaves. The clumping factor or the spatial structure of trees or crowns therefore played an important role in the corresponding studies. However, model sensitivity to these parameters decreased with stand density, stand LAI and leaf inclination (Kim et al., 2011).

Parameters of crown architecture played a secondary role. Only a few studies reported that changes in values of branch area, foliage inclination, and vertical distribution of leaves 
Table 5. Reported model evaluations.

\begin{tabular}{|c|c|c|}
\hline Plantation & Even-aged pure stand & Uneven-aged or mixed stands \\
\hline \multicolumn{3}{|l|}{ 1D Canopy } \\
\hline & No model name & \\
\hline & Govind et al. (2013) & \\
\hline & $\mathrm{R}^{2}=0.93$ & \\
\hline & $\mathrm{RMSE}=30-70 \mu \mathrm{mol} \mathrm{m}^{-2} \mathrm{~s}^{-1}$ & \\
\hline & no model name & \\
\hline & Kim et al. (2011) & \\
\hline & $\mathrm{RMSE}=0.06-135 \mu \mathrm{mol} \mathrm{m}{ }^{-2} \mathrm{~s}^{-1}$ & \\
\hline & 4C-A-RTM & \\
\hline & Bertin et al. (2011) & \\
\hline & $\begin{array}{l}\text { good relationship }\left(\mathrm{R}^{2}=0.92\right) \text { with } \\
\text { seedling growth }\end{array}$ & \\
\hline MIXLIGHT & MIXLIGHT & SORTIE \\
\hline Pinno et al. (2001) & Stadt and Lieffers (2000) & Canham et al. (1999) \\
\hline $\mathrm{R}^{2}=0.92$ & $\mathrm{R}^{2}=0.74^{*}$ & $\mathrm{R}^{2}=0.86$ \\
\hline \multicolumn{3}{|l|}{ 3D crown with quadric shapes } \\
\hline SORTIE-ND & SORTIE-ND & SILVI-STAR \\
\hline Paquette et al. (2008) & Boivin et al. (2011) & Koop and Sterck (1994) \\
\hline $\mathrm{R}^{2}=0.92$ & $\mathrm{R}^{2}=0.52$ & $\mathrm{R}^{2}=0.77-0.94$ \\
\hline SORTIE-ND & & SAMSARA \\
\hline Boivin et al. (2011) & & Courbaud et al. (2003) \\
\hline \multirow[t]{10}{*}{$\mathrm{R}^{2}=0.92$} & & $\mathrm{RMSE}=5.2 \%$ \\
\hline & & OLTREE \& SolTran \\
\hline & & Mariscal et al. (2004) \\
\hline & & $\mathrm{R}^{2}=0.94 *$ \\
\hline & MAESTRO & tRAYci \\
\hline & Wang and Jarvis (1990) & Gersonde et al. (2004) \\
\hline & $\mathrm{RMSE}=10 \%$ & $\mathrm{R}^{2}=0.80$ \\
\hline & TASS/tRAYci & CORONA \\
\hline & Brunner (1998) & Groot (2004) \\
\hline & $\mathrm{R}^{2}=0.97$ & $\mathrm{R}^{2}=0.88$ \\
\hline \multicolumn{3}{|l|}{ 3D crown with degenerated shapes } \\
\hline & tRAYci & $\mathrm{M} \mu \mathrm{SLIM}$ \\
\hline & Piboule (2001) & Da Silva et al. (2012) \\
\hline & $\mathrm{RMSE}=2 \%$ & $\begin{array}{l}\text { "in the range of model using } \\
\text { similar scales of description" }\end{array}$ \\
\hline & FOREST & FOREST \\
\hline & Cescatti (1997a) & Law et al. (2001) \\
\hline & $\mathrm{R}^{2}=0.97$ & $\mathrm{R}^{2}=0.62$ \\
\hline \multicolumn{3}{|c|}{ 3D surfaces of leaves, branches, and stems } \\
\hline AmapSim & no model name & \\
\hline Leroy et al. (2009) & Fournier et al. (1996) & \\
\hline no statistical difference in $3 / 4$ sites & $\mathrm{RMSE} \approx 10-15 \%$ & \\
\hline \multicolumn{3}{|l|}{ RATP } \\
\hline \multicolumn{3}{|l|}{ Sinoquet et al. (2001) } \\
\hline $\mathrm{RMSE}=60-120 \mu \mathrm{mol} \mathrm{m}^{-2} \mathrm{~s}^{-1}$ & & \\
\hline
\end{tabular}

Note: Models are classified by canopy model and type of forest stand forest structure. $\mathrm{R}^{2}$ with an asterisk $(*)$ means that the validation was performed with the predictions and measurements averaged between different sites rather than by point-to-point comparisons. 
Table 6. FRTM sensitivity to calibration parameters. For each parameter, we counted the number of publications (n) analyzing its effect on model predictions. According to the authors' conclusion, we classified their results into three categories: low, medium or high impact. The score corresponds to (high $+0.5 \cdot$ medium) $/ \mathrm{n}$.

\begin{tabular}{|c|c|c|c|c|c|c|}
\hline Parameter & $\mathrm{n}$ & low & medium & high & Score & References \\
\hline \multicolumn{7}{|l|}{ 3D crown model } \\
\hline crown radius & 7 & & & 7 & 1 & $\begin{array}{l}\text { Beaudet and Messier (2002); Brunner (1998); Gersonde et al. (2004); Pi- } \\
\text { boule (2001); Cescatti (1997); Da Silva et al. (2012); Stadt and Lieffers } \\
\text { (2000) }\end{array}$ \\
\hline crown shape & 2 & & & 2 & 1 & Brunner (1998); Piboule (2001) \\
\hline stand density & 2 & & & 2 & 1 & Bartelink (1998b); Essery et al. (2008) \\
\hline stand edge & 1 & & & 1 & 1 & Piboule (2001) \\
\hline understory vegetation & 1 & & & 1 & 1 & Beaudet and Messier (2002) \\
\hline tree height & 2 & & 1 & 1 & 0.8 & Beaudet and Messier (2002); Gersonde et al. (2004) \\
\hline crown length & 3 & & 2 & 1 & 0.7 & Piboule (2001); Stadt and Lieffers (2000); Gersonde et al. (2004) \\
\hline crown openness & 2 & 1 & & 1 & 0.5 & Beaudet and Messier (2002); Boivin et al. (2011) \\
\hline LAD & 5 & 2 & 2 & 1 & 0.4 & $\begin{array}{l}\text { Bartelink (1998b); Brunner (1998); Gersonde et al. (2004); Piboule } \\
\text { (2001); Stadt and Lieffers (2000) }\end{array}$ \\
\hline foliage clustering within crowns & 2 & 1 & 1 & & 0.3 & Bartelink (1998b); Cescatti (1997) \\
\hline trunk interception & 2 & 1 & 1 & & 0.25 & Brunner (1998); Fournier et al. (1996) \\
\hline crown max-width height & 1 & 1 & & & 0 & Piboule (2001) \\
\hline foliage clustering around shoots & 1 & 1 & & & 0 & Bartelink (1998b) \\
\hline foliage inclination & 1 & 1 & & & 0 & Stadt and Lieffers (2000) \\
\hline foliage as a sub-shell of crown & 1 & 1 & & & 0 & Gersonde et al. (2004); Cescatti (1997) \\
\hline \multicolumn{7}{|l|}{ 1D canopy model } \\
\hline leaf distribution & 1 & 1 & & & 0 & Wang and Jarvis (1990) \\
\hline crown surface & 1 & & & 1 & 1 & Duursma and Mäkelä (2007) \\
\hline leaf area & 3 & & & 3 & 1 & Kim et al. (2011); Bertin et al. (2011); Duursma and Mäkelä (2007) \\
\hline tree clumping & 3 & & 1 & 2 & 0.8 & Kim et al. (2011); Bertin et al. (2011); Duursma and Mäkelä (2007) \\
\hline branch area & 2 & & 2 & & 0.5 & Kim et al. (2011); Bertin et al. (2011) \\
\hline foliage inclination & 1 & & 1 & & 0.5 & Kim et al. (2011) \\
\hline vertical distribution of leaves & 1 & & 1 & & 0.5 & Kim et al. (2011) \\
\hline stem area & 2 & 1 & 1 & & 0.3 & Kim et al. (2011); Bertin et al. (2011) \\
\hline crown shape & 1 & 1 & & & 0 & Duursma and Mäkelä (2007) \\
\hline
\end{tabular}

affected seriously 1D model predictions. Similarly, crown shape and modeling stem interception had only a minor effect on model prediction.

\subsection{D crown model}

3D crown models appeared highly sensitive to the crown radius in all of the sensitivity analyses that studied the influence of this parameter (Beaudet et al., 2002; Brunner, 1998; Canham et al., 1994; Cescatti, 1997; Piboule, 2001; Stadt and Lieffers, 2000). It even appeared that estimating the crown radius using allometric relationships instead of measuring this parameter in the field individually for each tree significantly decreased model precision (Da Silva et al., 2012; Piboule, 2001). Indeed, crown dimensions largely depend on neighboring competition and tree history which are two effects usually not included in allometric relationships and that should be taken into account (Piboule et al., 2005).

Crown shape, tree height and crown base height seemed to have less influence (Brunner, 1998; Da Silva et al., 2012). FRTMs appeared highly sensitive to these parameters in less than $50 \%$ of the sensitivity analyses. For instance, Brunner (1998) tested two representations of crowns (with cones or asymmetric ellipsoids) and concluded that the two models gave similar results. Similarly, the height of maximum crown extension is needed to define the most complicated crown shapes and using such complex shape led to only minor improvements (Piboule, 2001).

The density of leaves and branches within crowns (expressed by LAD and crown openness) moderately affected the estimates of transmitted light beneath the forest canopy (Bartelink, 1998b; Brunner, 1998; Gersonde et al., 2004; Lieffers et al., 1999; Piboule, 2001; Stadt and Lieffers, 2000). Interestingly, reasonable changes in LAD affected model predictions markedly in only one of the five corresponding sensitivity analyses (Bartelink, 1998b; Brunner, 1998). It is worth noting that good estimates of LAD (3D-TM) or crown openness (3D-PE) require the correct delimitation of crown volume. Errors in computing the crown volume would lead to, for example, errors in calculating total leaf area. Similarly, LAD, clumping factors and extinction coefficients are strongly correlated (eq. 1, Bartelink (1998b)). This finding therefore justifies using fixed values of LAD per species.

Foliage distribution, clustering and inclination were studied to a slightly lesser extent, but these parameters appeared to have a small effect on 3D crown model predictions in forest stands (Bartelink, 1998b; Cescatti, 1997; Gersonde et al., 2004; Stadt and Lieffers, 2000; Wang and Jarvis, 1990). Foliage distribution likely depends on species. For instance, Cescatti (1997) reported evidence of the aggregation of spruce needles around vertices. Nevertheless, the influence of this 
aggregation on FRTM predictions appeared weak (Bartelink, 1998b; Cescatti, 1997), probably because 3D models are used mostly for stands with rather open canopy where gaps between crowns are more important than gaps within crowns. Similarly, modeling trunk interception was reported without significant effects in the two sensitivity analyses studying this parameter (Brunner, 1998; Fournier et al., 1996). With 3D crown model, using the default values of $k=0.5$ and $\Omega=1$ appeared therefore a reasonable simplification.

\section{Discussion}

This review illustrates and provides an overview of the variety of approaches used to represent a forest canopy and to estimate radiation intercepted by the canopy elements (Fig. 3). Even though more thoroughgoing conclusions could likely have been formulated with benchmarking of a wide set of FRTMs using a common data set with different stand structures, we were able to highlight the specificity of forest canopy in comparison with many agricultural crops and consequences in terms of modeling. The recent studies that we reviewed enabled us to quantify model uncertainty, to identify sensitive parameters, and to recommend the modeling approaches that best suit the different FRTM applications.

\subsection{Model uncertainty}

1D models accurately predict the temporal variations of irradiance (RMSE ranged roughly between 30 and $120 \mu \mathrm{mol} \mathrm{m}^{-2} \mathrm{~s}^{-1}$ ) within homogeneous stands (Govind et al., 2013; Kim et al., 2011). The vertical gradient of transmitted light beneath the canopy can be predicted reasonably well with multilayer models (Landsberg and Sands, 2010), but, the spatial variation in irradiance in the understory is not predicted by 1D models.

$3 \mathrm{D}$ crown models accurately predict averaged measures of transmitted light in a forest plot. The prediction of transmittance at a particular location beneath the canopy is clearly less accurate with errors often up to $20 \%$ of transmittance (Brunner, 1998; ?; Stadt and Lieffers, 2000). 3D crown models are capable of capturing a certain spatial variability of transmitted light but not with a resolution as precise as the one using light sensors. Within forest dynamic models, small errors in the spatial distribution of understory light should be considered as being of minor importance compared with, for example, the prediction of the cumulated area of microsites favorable to regeneration growth, i.e. receiving an irradiance within the range corresponding to the needs of a given species.

Moreover, model uncertainty increases with the complexity of the canopy. Transmittance beneath young plantation is better predicted than beneath uneven-aged and mixed stands. Transmitted light under homogeneous canopy is usually well predicted with every modeling approach. On the contrary, transmitted light under heterogeneous canopy is supposed to be hardly predicted with $1 \mathrm{D}$ models and to the best of our knowledge, no 1D model has furthermore been evaluated in heterogeneous mature stands. 3D crown model appeared so far the best solution to model the radiative transfer through such complex canopies.

Model uncertainty also decreases with increasing length of the simulated period especially if direct radiations are taken into account. As already pointed out for forest productivity models (Landsberg and Sands, 2010), predicting transmitted radiation for a brief period (e.g. $30 \mathrm{~min}$ ) requires very accurate radiation and canopy measurements. Accurate monthly and yearly averaged predictions are however more easily obtained (Essery et al., 2008; Landsberg and Sands, 2010), probably because of errors compensation.

We found no clear evidence regarding the advantage of using the turbid medium approach (3D-TM) versus the porous envelope approach (3D-PE). Both methods provided satisfactory results. Besides, if the crown openness varies with crown dimensions, both method are somehow related because $k$. LAD can be deduced from $p$ (Da Silva et al., 2012). If LAD and $p$ are species constant, then for every tree, independent of their size, the porous envelop approach affects transmittance to every crown in the same way, whereas it depends on crown size and beam orientation with the turbid medium approach. In this latter case, model performance of both approaches likely differs with the variability of crown dimensions.

\subsection{Sensitive input parameters}

Calibration efforts must focus primarily in the description of between-crown gaps or crown radius, which are the most sensitive parameters of FRTMs. This result is perhaps not intuitive but probably reflects that the forest canopy consist of deep crowns with dense foliage. Therefore, crowns intercept a large amount of light whatever their specific features. As a result, the proportion of transmitted light that is not intercepted by tree crowns, i.e. transmitted through gaps between crowns, is fundamental.

With 1D models, the key calibration parameters are the crown surface and (or) the LAI, which might further be corrected with extinction and clumping factors. On the other hand, 3D models require overall an accurate tree map and individual measurements of the crown radius, especially in order to characterize heterogeneous canopies. Crown shape appeared of lesser importance than crown radius and we did not find that it was worth using complex crown shapes. Furthermore, standard values of the extinction coefficient and clumping factors ( $k=0.5$ and $\Omega=1$, respectively) were often used with $3 \mathrm{D}$ crown model and frequently gave a good approximation. Therefore 3D models appear better suited for long simulation periods than 1D model because 1D model usually does not imply predicting changes of extinction and clumping factors.

\subsection{Applications}

We identified the advantages and drawbacks of the FRTM modeling approaches which allowed us to identify the approaches, that best suit to different potential applications of FRTM (Table 7). Overall, the potential applications depend mainly on how the canopy is modeled. 
Table 7. Synthesis of model advantages and drawbacks with suggested scale of uses, forest structures, and examples of applications.

\begin{tabular}{|c|c|c|c|c|c|}
\hline Model & Scale of uses & Forest structure & Advantages & Disadvantages & Applications \\
\hline 1D model & $\begin{array}{l}\text { from stand to } \\
\text { global, from } \\
\text { hours to one } \\
\text { year }\end{array}$ & $\begin{array}{l}\text { even-aged } \\
\text { stand, with one } \\
\text { or two species }\end{array}$ & $\begin{array}{l}\text { low number of field } \\
\text { data; easy to couple } \\
\text { to stand level data and } \\
\text { models (canopy gas } \\
\text { transfers, canopy cover, } \\
\text { Leaf Area Index). }\end{array}$ & $\begin{array}{l}\text { model parameters (fo- } \\
\text { liage distribution) are } \\
\text { hardly measured in the } \\
\text { field; lack of knowl- } \\
\text { edge to model ade- } \\
\text { quately changes of fo- } \\
\text { liage distribution param- } \\
\text { eters through time and } \\
\text { after thinning; do not } \\
\text { take explicitly into ac- } \\
\text { count spatial structure. }\end{array}$ & $\begin{array}{l}\text { net primary production; } \\
\text { carbon uptake; nutrient } \\
\text { cycling; photosynthesis } \\
\text { rate }\end{array}$ \\
\hline $3 \mathrm{D}-\mathrm{M}$ or $3 \mathrm{D}-\mathrm{PE}$ & $\begin{array}{l}\text { from plot to } \\
\text { stand, years }\end{array}$ & $\begin{array}{l}\text { even-aged or } \\
\text { unven-aged } \\
\text { stand, pure and } \\
\text { mixed stands }\end{array}$ & 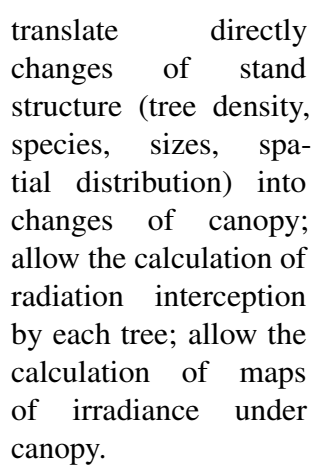 & $\begin{array}{l}\text { require information on } \\
\text { tree localization (ob- } \\
\text { tained either from field } \\
\text { measures or from the- } \\
\text { oretical distributions); } \\
\text { require information on } \\
\text { crown characteristics } \\
\text { (obtained either from } \\
\text { field measures or from } \\
\text { allometric relations) }\end{array}$ & $\begin{array}{l}\text { timber production; un- } \\
\text { derstory growth; forest } \\
\text { dynamic }\end{array}$ \\
\hline $3 \mathrm{D}-\mathrm{S}$ & $\begin{array}{l}\text { from leaf to tree, } \\
\text { from hours to } \\
\text { years }\end{array}$ & $\begin{array}{l}\text { isolated tree, } \\
\text { agroforestry } \\
\text { system, Ho- } \\
\text { mogeneous } \\
\text { stand }\end{array}$ & $\begin{array}{l}\text { allow the most precise } \\
\text { modelling of radiation } \\
\text { interception; can be cou- } \\
\text { pled to plant structure- } \\
\text { function models. }\end{array}$ & $\begin{array}{l}\text { require information on } \\
\text { plant architecture (ei- } \\
\text { ther measured or mod- } \\
\text { elled); high computing } \\
\text { complexity limiting the } \\
\text { number of simulated } \\
\text { trees. }\end{array}$ & $\begin{array}{l}\text { tree physiology; tree } \\
\text { morphogenesis; tree ar- } \\
\text { chitecture }\end{array}$ \\
\hline
\end{tabular}

1D models predict well the irradiance of canopy with a low number of input data and parameters. They are sensitive to the parameters describing foliage density and distribution ( $k$ and $\Omega$, respectively). They are ideally coupled with process-based of photosynthesis rate in order to explore forest productivity, carbon uptake, or nutrient cycling at the stand or greater scale. They are, however, limited to stands with one or two species and a relatively homogeneous (even-aged) structure. Moreover, the simulated forest should have a stable structure or the simulated time period should be limited because the calibration parameter depends strongly on stand structure and composition. In most cases, 1D models hardly predict light conditions after silvicultural operations such as partial harvests. Silvicultural operations often modify not only stand height, and density but also foliage features and distribution because some categories of trees are preferentially cut or because gaps are created. Through time, tree growth, self-thinning and self-pruning additionally modify the spatial distribution of foliage. In particular, as trees become mature, foliage tends to accumulate in fewer and bigger crowns. Next, as several regeneration cohorts develop, foliage becomes aggregated in crowns of varied dimensions.

3D crown models (3D-TM and 3D-PE) are better used in studies focusing on timber production and stand dynamics because, in many cases, those processes are generally not explicitly related to photosynthesis, transpiration, or nutrient cycling. They accommodate to heterogeneous stand structure. They include explicitly stand spatial structure, which enables studying the impact of forest structure (e.g. testing different silvicultural scenarios) on forest production and yield. This is an interesting feature with the general increasing interest devoted to uneven aged, shelterwood or close-to-nature silviculture in many countries. Moreover, single tree based models such as 3D crown models offer the best opportunity to analyze forest management strategies (Porte and Bartelink, 2002). Indeed, partial harvest modifies the average dimensions of trees and stand spatial structure. The increase in transmitted light is therefore complex and does not depend only on changes of stand density. 3D crown models are sensitive to canopy spatial structure and, hence, require precise mapping and crown mea- 


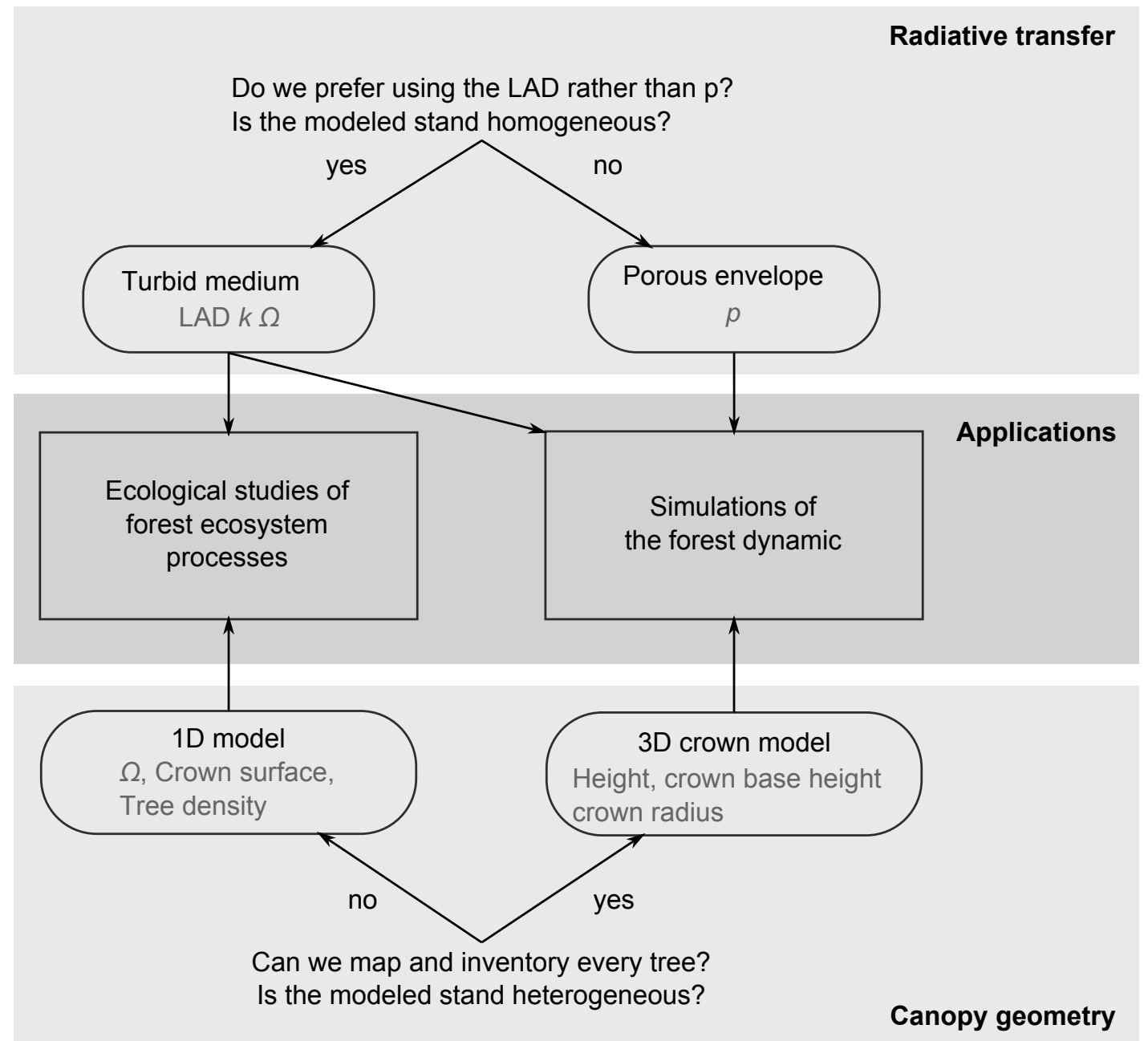

Figure 3. Overview of FRTM models according to their end-uses. Most FRTMs included in stand dynamic models are 3D crown models, whereas most FRTMs used for ecophysiological studies are 1D models. LAD, leaf area density; $k$, extinction coefficient; $\Omega$, clumping factor; $p$, crown openness; $1 \mathrm{D}$, one dimensional; $3 \mathrm{D}$, three-dimensional.

suring. Currently, the cost of these measurements limits the size of the modeled stand but the use of airborne technologies is promising (Essery et al., 2008).

3D surface models require acquiring large amounts of field data to reproduce tree architecture but they enable studying light capture at plant organ to tree levels (Rey et al., 2008). Whenever trees can realistically be duplicated in a forest model, 3D surface models offer good opportunity for studying the effects of tree architecture on tree physiological processes. Future technologies, such as terrestrial LiDAR can also help in acquiring the field data required to build 3D surface models for more complex stands.

\section{Acknowledgements}

This study was partly funded by the FNRS with the Research Fellow grant awarded to G. Ligot. We are grateful to Daniel Kneeshaw for his careful proofreading of the manuscript and his constructive comments.

\section{References}

Astrup, R. and Larson, B. C. Regional variability of speciesspecific crown openness for aspen and spruce in western boreal canada. For. Ecol. Manage., 228(1-3):241-250, 2006. doi: 10.1016/j.foreco.2006.02.048.

Aubin, I., Beaudet, M., and Messier, C. Light extinction coefficients specific to the understory vegetation of the southern boreal forest, quebec. Can. J. For. Res., 30(1): 168-177, 2000. doi: 10.1139/x99-185.

Balandier, P., Collet, C., Miller, J. H., Reynolds, P. E., and Zedaker, S. M. Designing forest vegetation management strategies based on the mechanisms and dynamics of crop tree competition by neighbouring vegetation. Forestry, 79 (1):3-27, 2006. doi: 10.1093/forestry/cpi056.

Balandier, P., Sinoquet, H., Frak, E., Giuliani, R., Vandame, M., Descamps, S., Coll, L., Adam, B., Prevosto, B., and 
Curt, T. Six-year time course of light-use efficiency, carbon gain and growth of beech saplings (fagus sylvatica) planted under a scots pine (pinus sylvestris) shelterwood. Tree Physiol., 27(8):1073-1082, 2007. doi: 10.1093/treephys/27.8.1073.

Balandier, P., Marquier, A., Gaudio, N., Wehrlen, L., Casella, E., Coll, L., Kiewitt, A., and Harmer, R. Methods for describing light capture by understorey weeds in temperate forests: consequences for tree regeneration. In Bentsen, N., editor, Final COST E47 Conference, Forest vegetation management towards environmental sustainability, pages 73-75, Year.

Barbier, S., Gosselin, F., and Balandier, P. Influence of tree species on understory vegetation diversity and mechanisms involved - a critical review for temperate and boreal forests. For. Ecol. Manage., 254(1):1-15, 2008. doi: 10.1016/j.foreco.2007.09.038.

Bartelink, H. H. A model of dry matter partitioning in trees. Tree Physiol., 18(2):91-101, 1998a. doi: 10.1093/treephys/18.2.91.

Bartelink, H. H. Radiation interception by forest trees: a simulation study on effects of stand density and foliage clustering on absorption and transmission. Ecol. Model., 105(2-3): 213-225, 1998b. doi: 10.1016/S0304-3800(97)00165-8.

Beaudet, M. and Messier, C. Variation in canopy openness and light transmission following selection cutting in northern hardwood stands: an assessment based on hemispherical photographs. Agric. For. Meteorol., 110(3):217-228, 2002. doi: 10.1016/S0168-1923(01)00289-1.

Beaudet, M., Messier, C., and Canham, C. D. Predictions of understorey light conditions in northern hardwood forests following parameterization, sensitivity analysis, and tests of the sortie light model. For. Ecol. Manage., 165(1-3): 235-248, 2002. doi: 10.1016/S0378-1127(01)00621-1.

Beaudet, M., Harvey, B. D., Messier, C., Coates, K. D., Poulin, J., Kneeshaw, D. D., Brais, S., and Bergeron, Y. Managing understory light conditions in boreal mixedwoods through variation in the intensity and spatial pattern of harvest: a modelling approach. For. Ecol. Manage., 261(1):84-94, 2011. doi: 10.1016/j.foreco.2010.09.033.

Bertin, S., Palmroth, S., Kim, H. S., Perks, M. P., Mencuccini, M., and Oren, R. Modelling understorey light for seedling regeneration in continuous cover forestry canopies. Forestry, 84(4):397-409, 2011. doi: 10.1093/forestry/cpr026.

Boivin, F., Paquette, A., Racine, P. B., and Messier, C. A fast and reliable method for the delineation of tree crown outlines for the computation of crown openness values and other crown parameters. Can. J. For. Res., 41(9):18271835, 2011. doi: 10.1139/x11-107.
Breda, N. Ground-based measurements of leaf area index: a review of methods, instruments and current controversies. J. Exp. Bot., 54(392):2403-2417, 2003. doi: $10.1093 / \mathrm{jxb} / \mathrm{erg} 263$.

Brunner, A. A light model for spatially explicit forest stand models. For. Ecol. Manage., 107(1-3):19-46, 1998. doi: 10.1016/S0378-1127(97)00325-3.

Campbell, G. S. and Norman, J. M. An introduction to environmental biophysics. Springer, New-York, 1998. doi: 10.1007/978-1-4612-1626-1.

Canham, C. D., Finzi, A. C., Pacala, S. W., and Burbank, D. H. Causes and consequences of resource heterogeneity in forests - interspecific variation in light transmission by canopy trees. Can. J. For. Res., 24(2):337-349, 1994. doi: 10.1139/x94-046.

Canham, C. D., Coates, K. D., Bartemucci, P., and Quaglia, S. Measurement and modeling of spatially explicit variation in light transmission through interior cedar-hemlock forests of british columbia. Can. J. For. Res., 29(11):1775-1783, 1999. doi: 10.1139/x99-151.

Cescatti, A. Modelling the radiative transfer in discontinuous canopies of asymmetric crowns. i. model structure and algorithms. Ecol. Model., 101(2-3):263-274, 1997. doi: 10.1016/S0304-3800(97)00050-1.

Chen, J. M., Blanken, P. D., Black, T. A., Guilbeault, M., and Chen, S. Radiation regime and canopy architecture in a boreal aspen forest. Agric. For. Meteorol., 86(1-2):107-125, 1997. doi: 10.1016/S0168-1923(96)02402-1.

Chen, J. M., Liu, J., Cihlar, J., and Goulden, M. L. Daily canopy photosynthesis model through temporal and spatial scaling for remote sensing applications. Ecol. Model., 124 (2-3):99-119, 1999. doi: 10.1016/S0304-3800(99)00156-8.

Courbaud, B., Goreaud, F., Dreyfus, P., and Bonnet, F. R. Evaluating thinning strategies using a tree distance dependent growth model: some examples based on the capsis software "uneven-aged spruce forests" module. For. Ecol. Manage., 145(1-2):15-28, 2001. doi: 10.1016/S03781127(00)00571-5.

Courbaud, B., de Coligny, F., and Cordonnier, T. Simulating radiation distribution in a heterogeneous norway spruce forest on a slope. Agric. For. Meteorol., 116(1-2):1-18, 2003. doi: 10.1016/S0168-1923(02)00254-X.

Da Silva, D., Boudon, F., Godin, C., and Sinoquet, H. Multiscale framework for modeling and analyzing light interception by trees. Multiscale Model. Simul., 7(2):910-933, 2008. doi: 10.1137/08071394X.

Da Silva, D., Balandier, P., Boudon, F., Marquier, A., and Godin, C. Modeling of light transmission under heterogeneous forest canopy: an appraisal of the effect of the 
precision level of crown description. Ann. For. Sci., 69(2): 191-193, 2012. doi: 10.1007/s13595-011-0139-2.

Dauzat, J. and Eroy, M. N. Simulating light regime and intercrop yields in coconut based farming systems. Eur. J. Agron., 7(1-3):63-74, 1997 doi: 10.1016/S11610301(97)00029-4.

Duursma, R. A. and Mäkelä, A. Summary models for light interception and light-use efficiency of non-homogeneous canopies. Tree Physiol., 27(6):859-870, 2007. doi: 10.1093/treephys/27.6.859.

Essery, R., Bunting, P., Rowlands, A., Rutter, N., Hardy, J., Melloh, R., Link, T., Marks, D., and Pomeroy, J. Radiative transfer modeling of a coniferous canopy characterized by airborne remote sensing. J. Hydrometeor., 9(2):228-241, 2008. doi: 10.1175/2007jhm870.1.

Fournier, R. A., Landry, R., August, N. M., Fedosejevs, G., and Gauthier, R. P. Modelling light obstruction in three conifer forests using hemispherical photography and fine tree architecture. Agric. For. Meteorol., 82(1-4):47-72, 1996. doi: 10.1016/0168-1923(96)02345-3.

Galen, C., Rabenold, J. J., and Liscum, E. Functional ecology of a blue light photoreceptor: effects of phototropin-1 on root growth enhance drought tolerance in arabidopsis thaliana. New Phytol., 173(1):91-99, 2007. doi: 10.1111/j.1469-8137.2006.01893.x.

Gersonde, R., Battles, J. J., and O'Hara, K. L. Characterizing the light environment in sierra nevada mixed-conifer forests using a spatially explicit light model. Can. J. For. Res., 34 (6):1332-1342, 2004. doi: 10.1139/x04-013.

Goudriaan, J. Crop micrometeorology: a simulation study. $\mathrm{PhD}$ thesis, 1977.

Govind, A., Guyon, D., Roujean, J.-L., Yauschew-Raguenes, N., Kumari, J., Pisek, J., and Wigneron, J.-P. Effects of canopy architectural parameterizations on the modeling of radiative transfer mechanism. Ecol. Model., 251:114-126, 2013. doi: 10.1016/j.ecolmodel.2012.11.014.

Groot, A. A model to estimate light interception by tree crowns, applied to black spruce. Can. J. For. Res., 34(4): 788-799, 2004. doi: 10.1139/x03-242.

Jarvis, P. G. and Leverenz, J. W. Productivity of temperate, deciduous and evergreen forests, volume 12, chapter 8, pages 234-280. Springer, Berlin, 1983. doi: 10.1007/9783-642-68156-1'9.

Jonckheere, I., Fleck, S., Nackaerts, K., Muys, B., Coppin, P., Weiss, M., and Baret, F. Review of methods for in situ leaf area index determination - part i. theories, sensors and hemispherical photography. Agric. For. Meteorol., 121(1-2): 19-35, 2004. doi: 10.1016/j.agrformet.2003.08.027.
Kim, H. S., Palmroth, S., Thérézien, M., Stenberg, P., and Oren, R. Analysis of the sensitivity of absorbed light and incident light profile to various canopy architecture and stand conditions. Tree Physiol., 31(1):30-47, 2011. doi: 10.1093/treephys/tpq098.

Koop, H. and Sterck, F. J. Light penetration through structurally complex forest canopies - an example of a lowland tropical rain-forest. For. Ecol. Manage., 69(1-3):111-122, 1994. doi: 10.1016/0378-1127(94)90223-2.

Kuuluvainen, T. and Pukkala, T. Interaction between canopy architecture and photosynthetically active direct radiation at different latitudes: simulation experiments and their ecological implications. L'arbre. Biologie et développement., h.s.:277-291, 1991.

Kuusk, A. Simulation of crop reflectance (including hot spot effect), pages 263-270. INRA, Lusignan, France, 1993.

Landsberg, J. J. and Sands, P. Physiological ecology of forest production: principles, processes and models, volume 4. Academic Press, 2010.

Law, B. E., Cescatti, A., and Baldocchi, D. D. Leaf area distribution and radiative transfer in open-canopy forests: implications for mass and energy exchange. Tree Physiol., 21(12-13):777-787, 2001. doi: 10.1093/treephys/21.1213.777.

Leroy, C., Sabatier, S., Wahyuni, N., Barczi, J.-F., Dauzat, J., Laurans, M., and Auclair, D. Virtual trees and light capture: a method for optimizing agroforestry stand design. Agroforest. Syst., 77(1):37-47, 2009. doi: 10.1007/s10457009-9232-z.

Lieffers, V. J., Messier, C., Stadt, K. J., Gendron, F., and Comeau, P. G. Predicting and managing light in the understory of boreal forests. Can. J. For. Res., 29(6):796-811, 1999. doi: 10.1139/x98-165.

Mariscal, M. J., Martens, S. N., Ustin, S. L., Chen, J., Weiss, S. B., and Roberts, D. A. Light-transmission profiles in an old-growth forest canopy: simulations of photosynthetically active radiation by using spatially explicit radiative transfer models. Ecosystems, 7(5):454-467, 2004. doi: 10.1007/s10021-004-0137-4.

Messier, C., Doucet, R., Ruel, J. C., Claveau, Y., Kelly, C., and Lechowicz, M. J. Functional ecology of advance regeneration in relation to light in boreal forests. Can. J. For. Res., 29(6):812-823, 1999. doi: 10.1139/x99-070.

Moulia, B. and Sinoquet, H. Three-dimensional digitizing systems for plant canopy geometrical structure: a review, pages 183-193. INRA, Lusignan, France, 1993.

Niinemets, . A review of light interception in plant stands from leaf to canopy in different plant functional types and 
in species with varying shade tolerance. Ecol. Res., 25(4): 693-714, 2010. doi: 10.1007/s11284-010-0712-4.

Nock, C. A., Caspersen, J. P., and Thomas, S. C. Large ontogenetic declines in intra-crown leaf area index in two temperate deciduous tree species. Ecology, 89(3):744-753, 2008. doi: 10.1890/07-0531.1.

Pacala, S. W., Canham, C. D., Saponara, J., Silander, J. A., Kobe, R. K., and Ribbens, E. Forest models defined by field measurements: estimation, error analysis and dynamics. Ecol. Monogr., 66(1):1-43, 1996. doi: 10.2307/2963479.

Paquette, A., Messier, C., Perinet, P., and Cogliastro, A. Simulating light availability under different hybrid poplar clones in a mixed intensive plantation system. For. Sci., 54(5): 481-489, 2008.

Parker, G. G. Canopy structure and light environment of an old-growth douglas-fir/western hemlock forest. Northwest Sci., 71(4):261-270, 1997.

Piboule, A. Validation et analyse de sensibilité d'un modèle de transfert radiatif en vue de son application à la cartographie de l'éclairement en peuplement forestier. D.e.a., 2001.

Piboule, A., Collet, C., Frochot, H., and Dhote, J. F. Reconstructing crown shape from stem diameter and tree position to supply light models. i. algorithms and comparison of light simulations. Ann. For. Sci., 62(7):645-657, 2005. doi: 10.1051/forest:2005071.

Pierce, L. L. and Running, S. W. Rapid estimation of coniferous forest leaf area index using a portable integrating radiometer. Ecology, 69(6):1762-1767, 1988. doi: $10.2307 / 1941154$.

Pinno, B. D., Lieffers, V. J., and Stadt, K. J. Measuring and modelling the crown and light transmission characteristics of juvenile aspen. Can. J. For. Res., 31(11):1930-1939, 2001. doi: 10.1139/cjfr-31-11-1930.

Porte, A. and Bartelink, H. H. Modelling mixed forest growth: a review of models for forest management. Ecol. Model., 150(1-2):141-188, 2002. doi: 10.1016/S03043800(01)00476-8.

Pukkala, T., Kuuluvainen, T., and Stenberg, P. Below-canopy distribution of photosynthetically active radiation and its relation to seedling growth in a boreal pinus-sylvestris stand - a simulation approach. Scand. J. For. Res., 8(3):313-325, 1993. doi: 10.1080/02827589309382780.

Rey, H., Dauzat, J., Chenu, K., Barczi, J.-F., Dosio, G. A. A., and Lecoeur, J. Using a 3-d virtual sunflower to simulate light capture at organ, plant and plot levels: contribution of organ interception, impact of heliotropism and analysis of genotypic differences. Ann. Bot., 101(8):1139-1151, 2008. doi: $10.1093 / \mathrm{aob} / \mathrm{mcm} 300$.
Ross, J. The radiation regime and architecture of plant stands, volume 3 of Tasks for vegetation sciences. Springer, Boston, 1981. doi: 10.1007/978-94-009-8647-3.

Sinoquet, H., Varlet-Grancher, C., and Bonhomme, R. Modelling radiative transfer within homogeneous canopies : basic concepts, pages 207-228. INRA, Lusignan, France, 1993.

Sinoquet, H., Le Roux, X., Adam, B., Ameglio, T., and Daudet, F. A. Ratp: a model for simulating the spatial distribution of radiation absorption, transpiration and photosynthesis within canopies: application to an isolated tree crown. Plant Cell Environ., 24(4):395-406, 2001. doi: 10.1046/j.1365-3040.2001.00694.x.

Sinoquet, H., Sonohat, G., Phattaralerphong, J., and Godin, C. Foliage randomness and light interception in 3-d digitized trees: an analysis from multiscale discretization of the canopy. Plant Cell Environ., 28(9):1158-1170, 2005. doi: 10.1111/j.1365-3040.2005.01353.x.

Sprugel, D. G., Rascher, K. G., Gersonde, R., Dovciak, M., Lutz, J. A., and Halpern, C. B. Spatially explicit modeling of overstory manipulations in young forests: Effects on stand structure and light. Ecol. Model., 220(24):3565-3575, 2009. doi: 10.1016/j.ecolmodel.2009.07.029.

Stadt, K. J. and Lieffers, V. J. Mixlight: a flexible light transmission model for mixed-species forest stands. Agric. For. Meteorol., 102(4):235-252, 2000. doi: 10.1016/S01681923(00)00128-3.

Thérézien, M., Palmroth, S., Brady, R., and Oren, R. Estimation of light interception properties of conifer shoots by an improved photographic method and a $3 \mathrm{~d}$ model of shoot structure. Tree Physiol., 27(10):1375-1387, 2007. doi: 10.1093/treephys/27.10.1375.

Tian, H., Chen, G., Liu, M., Zhang, C., Sun, G., Lu, C., Xu, X., Ren, W., Pan, S., and Chappelka, A. Model estimates of net primary productivity, evapotranspiration, and water use efficiency in the terrestrial ecosystems of the southern united states during 1895-2007. For. Ecol. Manage., 259 (7):1311-1327, 2010. doi: 10.1016/j.foreco.2009.10.009.

Wang, Y. P. and Jarvis, P. G. Description and validation of an array model-maestro. Agric. For. Meteorol., 51(3): 257-280, 1990. doi: 10.1016/0168-1923(90)90112-J.

Webb, W. L. and Ungs, M. J. Three dimensional distribution of needle and stem surface area in a douglas-fir. Tree Physiol., 13(2):203-212, 1993. doi: 10.1093/treephys/13.2.203.

Weiss, M., Baret, F., Smith, G. J., Jonckheere, I., and Coppin, P. Review of methods for in situ leaf area index (lai) determination part ii. estimation of lai, errors and sampling. Agric. For. Meteorol., 121(1-2):37-53, 2004. doi: 10.1016/j.agrformet.2003.08.001. 
Williams, D. L. A comparison of spectral reflectance properties at the needle, branch, and canopy level for selected conifer species. Remote Sens. Environ., 35(2-3):79-93, 1991. doi: 10.1016/0034-4257(91)90002-N. 